\title{
Successful aortic valve repair for severe aortic insufficiency caused by radiofrequency ablation
}

\author{
Giovanni Melina, MD, PhD, ${ }^{a}$ Riccardo Codecasa, MD, ${ }^{\text {a }}$ Irene Capecchi, MD, ${ }^{a}$ Maria Luisa Gianfaldoni, MD, \\ Sergio Bevilacqua, MD, ${ }^{a}$ Carlo Sorbara, MD, ${ }^{a}$ and Pier Luigi Stefàno, MD, ${ }^{a}$ Firenze, Italy
}

$\mathrm{V}$ alvular lesions after left-sided radiofrequency catheter ablation with the retrograde aortic technique vary from $1 \%$ to $8 \%$ and are more common in younger patients. ${ }^{1,2}$ Mitral regurgitation is slightly more frequent than aortic regurgitation (AR), and in both cases lesions cause trivial or mild insufficiency. New-onset valvular lesions are mostly the result of catheter manipulations rather than tissue injury related to radiofrequency energy application. ${ }^{1} \mathrm{~A}$ case of aortic leaflet perforation was first described by Seifert and associates, ${ }^{3}$ but AR was only trivial. The present case report describes a patient who had severe AR after retrograde radiofrequency catheter ablation and underwent successful aortic valve repair. It is hoped that this information provides further insight into the follow-up and management of such a complication.

\section{Clinical Summary}

A 50-year-old woman was successfully treated for a WolffParkinson-White syndrome with catheter ablation of a left posterolateral accessory pathway by using a retrograde approach across the aortic valve. Moderate AR developed after the procedure. At strict follow-up, the insufficiency rapidly worsened causing severe dyspnea.

The transthoracic echocardiogram showed a normal aortic box with normal left ventricular dimensions and function. AR was quantified by a large diastolic high-velocity jet filling the left ventricular outflow tract and reaching the apex (pressure half-time $300 \mathrm{~ms}$ ). No other valvular defects were found. Diastolic mitral flow pattern showed impaired relaxation. In the long-axis view, the transesophageal echocardiogram showed a normal aortic root with evidence of a prolapse of the noncoronary cusp. In the short-axis view, the aortic valve appeared tricuspid with a normal opening and rupture of the free edge of

\footnotetext{
From the Dipartimento Cuore-Vasi, Azienda Ospedaliero-Universitaria Careggi, ${ }^{a}$ and Dipartimento di Cardiologia, Azienda Ospedaliera di Empoli, ${ }^{\text {b }}$ Firenze, Italy.

Received for publication Nov 9, 2004; accepted for publication Dec 10, 2004.

Address for reprints: Riccardo Codecasa, U.O. Cardiochirurgia, Azienda Ospedaliera Careggi, Viale Morgagni 85, 50139 Firenze, Italy (E-mail: riccardocodecasa@yahoo.com).

J Thorac Cardiovasc Surg 2005;130:564-5

$0022-5223 / \$ 30.00$

Copyright $\odot 2005$ by The American Association for Thoracic Surgery

doi:10.1016/j.jtcvs.2004.12.024
}

the noncoronary cusp causing an eccentric jet directed toward the septum. Figure 1 shows the preoperative assessment. The angiographic study confirmed the diagnosis of severe AR with good left ventricular function and no coronary artery lesions. She was then referred for urgent surgical treatment.

Operation findings and surgical technique. The operation was performed through a median sternotomy, aorta-right atrium cannulation, and Buckberg cardioplegia administration. The native aortic valve had a laceration of the free edge of the (normal) noncoronary leaflet, prolapsing into the left ventricle. The laceration was fixed with an interrupted 7-0 polypropylene suture, the free edge was plicated with a running polytetrafluoroethylene (Gore-Tex, W. L. Gore \& Associates, Inc., Flagstaff, Ariz) suture, and the commissures between the noncoronary and right coronary leaflet and between the noncoronary and left coronary leaflet were resuspended with interrupted 5-0 polypropylene sutures.

Intraoperative echocardiographic control. The intraoperative transesophageal echocardiogram showed good left ventricular function and no mitral regurgitation, and the aortic valve was continent with good excursion of the 3 leaflets.

Postoperative course. The postoperative period was uneventful, and the patient was discharged on the fifth postoperative day in sinus rhythm. At discharge the transthoracic echocardiographic assessment showed only trivial central AR, normal valve opening, and normal left ventricular dimensions and function. At the 6-month follow-up, she was symptom free and the echocardiogram remained the same (Figure 2).

\section{Comment}

We report this case as a useful tool for the management of similar situations. It is therefore mandatory to maintain strict clinical surveillance of patients who are catheter ablated for arrhythmias. Echocardiography may need to be performed routinely to detect any valve-related complications and possibly institute corrective measures. AR rarely occurs after radiofrequency ablation and is usually trivial or mild and a self-limiting complication. ${ }^{1-3}$ In this case, the patient had acute AR with rapid worsening of the symptoms. Therefore, the timing of operation in such circumstances is critical to avoid heart and systemic deterioration. When the valve anatomy is normal, except for the iatrogenic lesion of the cusp, either valve replacement or repair is possible. We believe the latter is better because of the anatomic and clinical issues. The absence of pathologic alterations of the leaflets may be reasonably predictive of a good result of the repair, either short or long term. From a clinical perspective, the relatively young age of the patient would have suggested a mechanical prosthesis for re- 

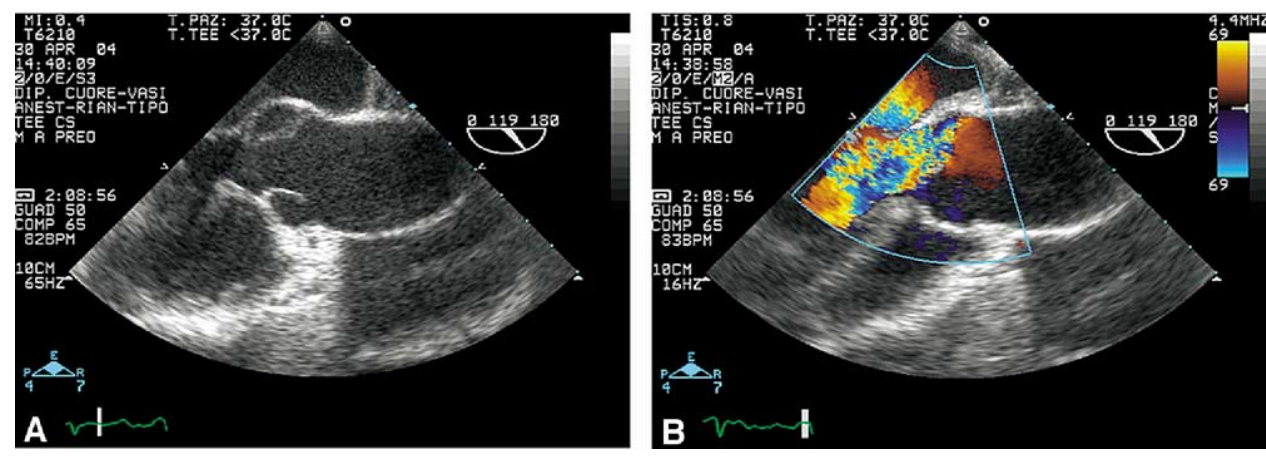

Figure 1. Preoperative transesophageal echocardiograph assessment.
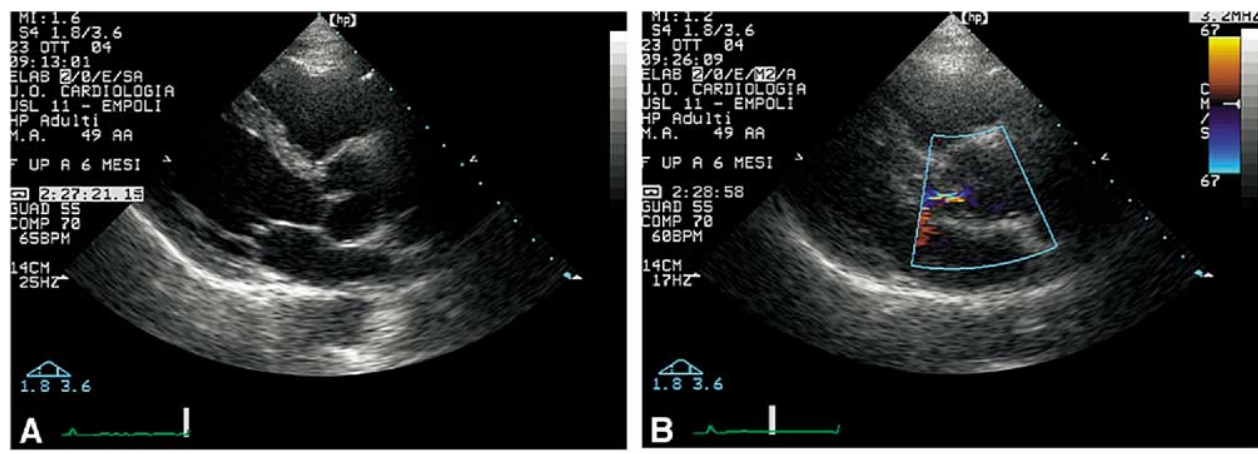

Figure 2. Transesophageal echocardiograph at the 6-month follow-up.

placement, with the anticoagulation-related problems. We suggest that aortic valve repair be performed in high-volume centers with a wide experience in valve-sparing techniques. In this patient valve repair was successful, and she had a prompt recovery after surgery. Early echocardiographic control showed normal valve function and good left ventricular function. Longterm results might be different for posttraumatic leaflet repair, and a specific follow-up would therefore be advisable. It is hoped that this single case provides insight for both clinicians and researchers.

\section{References}

1. Olsson A, Darpo B, Bergfeldt L, Rosenqvist M. Frequency and long term follow up of valvar insufficiency caused by retrograde aortic radiofrequency catheter ablation procedures. Heart. 1999;81:292-6.

2. Law IH, Fischbach PS, LeRoy S, Lloyd TR, Rocchini AP, Dick M. Access to the left atrium for delivery of radiofrequency ablation in young patients: retrograde aortic vs transseptal approach. Pediatr Cardiol. 2001;22:204-9.

3. Seifert MJ, Morady F, Calkins HG, Langberg JJ. Aortic leaflet perforation during radiofrequency ablation. Pacing Clin Electrophysiol. 1991;14:1582-5. 